\title{
A "quickscore" method for immunohistochemical semiquantitation: validation for oestrogen receptor in breast carcinomas
}

\author{
S Detre, G Saccani Jotti, M Dowsett
}

\begin{abstract}
Immunohistochemistry is increasingly used in the assessment of markers for breast cancer prognosis. Semiquantitation is frequently desirable but, other than by the use of image analysis, the approaches currently in use are cumbersome. The most common method used is the $H$-score which takes into consideration the staining intensity in conjunction with the percentage of cells staining positively in breast carcinoma tissue. A "quickscore" has been developed which dispenses with the need to count individual cells. The quantitative biochemical Abbott enzyme immunoassay (EIA) and the Dako immunohistochemical assay (IHA) incorporating a semiquantitative $\mathbf{H}$-score, have been used as standards against which the IHA quickscore for the semiquantitation of oestrogen receptor expression was tested. A good correlation was found between the quickscore and the EIA, which was as good as that between the H-score and EIA. The quickscore is a valid approach and there is no advantage in using the more rigorous $H$-score. $A$ positive cut off quickscore of $\geqslant 3$ has been suggested.

(f Clin Pathol 1995;48:876-879)
\end{abstract}

Keywords: Immunohistochemistry, quickscore, oestrogen receptor, breast cancer.

Oestrogen receptor determination is important in the study of the biology of breast cancers and for determining the clinical implications of hormone treatment. ${ }^{1}$ Recently, the immunohistochemical assay (IHA) has gained favour over the gold standard enzyme immunoassay (EIA) because it is cheaper, quicker and easier to perform and enables direct visualisation of malignant tumour receptor localisation in small tissue samples. ${ }^{2}$ One disadvantage of the immunohistochemical approach is the laborious $\mathrm{H}$-score procedure which is prone to a degree of discordance between assessors. ${ }^{3}$

As the immunohistochemical approach is gaining increasing support for routine assessment of oestrogen receptor expression, a more rapid scoring scheme would be useful especially when appraising large numbers of sections from clinical studies. To this end a quickscore method such as that suggested by Barnes et $a l^{4}$ would expedite matters. The method described by Barnes et al, however, involves the addition of scores for staining intensity and the proportion of positive cells, which is a departure from the $\mathrm{H}$-score which is based on multiplication of these components and intuitively is more appropriate if staining intensity reflects antigen concentration. The aim of this study was to assess whether a modification of the quickscore to a multiplicative form was valid and could provide an acceptable alternative to the H-score. Two extra categories for the proportion of cells staining positively were also included to permit a completely negative result, and to take into account the presence of very small numbers of positive cells.

\section{Methods}

Ninety six primary breast cancer surgical specimens, embedded in paraffin wax, were studied. These tumours were taken from a cohort of 119 untreated patients studied previously ${ }^{5}$ and the oestrogen receptor EIA, using the Abbott H222 antibody, and the oestrogen receptor IHA, using the Dako 1D5 antibody, have been described in detail. The reduction in sample size to 96 tumours was necessary because 17 mismatches had occurred between EIA and IHA pairs of results and in order not to confound the scoring appraisal these mismatches were excluded from this investigation. A further six tumours were excluded because full results were not available.

\section{SCORING ASSESSMENT}

For H-score assessment, 10 fields were chosen at random at $\times 400$ magnification and the staining intensity in the malignant cell nuclei was scored as $0,1,2$, or 3 corresponding to the presence of negative, weak, intermediate, and strong brown staining, respectively. The total number of cells in each field and the number of cells stained at each intensity were counted. The average percentage positive was calculated and the following formula ${ }^{3}$ was applied:

IHA H-score $=(\%$ of cells stained at intensity category $1 \times 1)+(\%$ of cells stained at intensity category $2 \times 2)+(\%$ of cells stained at intensity category $3 \times 3)$.

A $\mathrm{H}$-score between 0 and 300 was obtained where 300 was equal to $100 \%$ of tumour cells stained strongly $(3+)$.

The immunohistochemically stained sections were subsequently given a quickscore independently by two of the authors without prior consultation or recourse to clinical, bio- 
Table 1 Spearman rank correlation coefficients for comparisons of the various methods of oestrogen receptor measurement in breast carcinomas

\begin{tabular}{ll}
\hline Comparison of methods of measurement & rho \\
\hline Q $=$ A $\times$ B $v$ H score & $0 \cdot 892$ \\
Q $=$ A + B $v$ H score & $0 \cdot 887$ \\
H-score $v$ EIA & $0 \cdot 843$ \\
Q $=$ A $\times$ B EIA & $0 \cdot 831$ \\
Q $=$ A + B $v$ EIA & $0 \cdot 832$ \\
\hline
\end{tabular}

rho $_{\mathrm{t}}=$ regression coefficient corrected for ties; $Q=$ quickscore where $A=$ proportion of oestrogen receptor positive malignan cells and $\mathrm{B}=$ staining intensity. $\mathrm{p}<0.0001$

chemical or previously recorded $\mathrm{H}$-score data. The quickscore categories were based on both the intensity and the proportion of brown staining nuclei. In our modification the proportion of malignant cells staining positively throughout the section was termed category $\mathrm{A}$ and was assigned scores from 1 to $6(1=0-4 \% ; 2=$ $5-19 \% ; 3=20-39 \% ; 4=40-59 \% ; 5=60-79 \%$; $6=80-100 \%)$. The whole section was scanned at low power in order to gauge the general level of intensity throughout. The average intensity, corresponding to the presence of negative, weak, intermediate, and strong staining, was given a score from 0 to 3 , respectively, and termed category B. Category A was added to category $B(A+B)$ to form an additive quickscore and recorded in parallel with the product $(\mathrm{A} \times \mathrm{B})$ as a multiplicative quickscore. The results were tabulated and EIA and IHA results were compared with the quickscore using Spearman rank correlation statistics.

\section{Results}

The EIA values ranged from 0 to 597 fmoles $\mathrm{mg}$ protein and $62 \%$ of the tumours were oestrogen receptor positive (cut off $\geqslant 10 \mathrm{fmoles}$ / $\mathrm{mg}$ protein). H-scores ranged from 0 to 190. Additive and multiplicative quickscores covered the full range-that is, $(A+B)=1-9$ and $(A \times B)=0-18$, respectively. Table 1 shows a comparison of the Spearman rank correlations for the three oestrogen receptor methods and the individual categories of the $\mathrm{A} \times \mathrm{B}$ quickscore are compared with intervals of $\mathrm{H}$-scores and EIA in table 2. There was no difference between the additive and multiplicative quick- scores and a good positive correlation was observed between the IHA and the EIA (table 1). More importantly, the correlation between both of the quickscores and the EIA was very similar to that for the H-score and the EIA.

We also compared the performance of the two quickscore approaches with two separate assessors. Total agreement of score occurred in $73 \%$ of cases and demonstrated a very strong correlation between the multiplicative and additive quickscore $\left(\mathrm{rho}_{\mathrm{t}}=0.994\right)$. In the seven cases where disagreement occurred, five were at the highest score levels-for example, $A \times B=18$ and $15, A+B=8$ and 9 , and two at the lowest levels-for example, $\mathrm{A} \times \mathrm{B}=1$ and $2, \mathrm{~A}+\mathrm{B}=2$ and 3 . The maximum quickscore was attained in many cases, especially where high EIA values occurred. Interestingly, a very high $\mathrm{H}$-score level was never achieved.

\section{Discussion}

The concordance between the two observers indicates that both quickscores are reproducible although more studies from other laboratories are necessary to confirm this. The IHA quickscore compared favourably with the gold standard EIA. That the quickscore is of equivalent value to the $\mathrm{H}$-score, when using the same immunohistochemical technique, was confirmed by the good Spearman rank correlation between them.

The reason why these sections did not have very high $\mathrm{H}$-scores could have been because tumours in this cohort expressed oestrogen receptor at a low level. This, however, was not the case when the quickscore was applied as maximum scores were reported in $19 \%$ (GSJ) and $14 \%(\mathrm{SD})$ of cases.

The quickscore seems to be less prone to variation than the $\mathrm{H}$-score because the observer evaluates the whole section, estimating an overall impression of intensity when scoring and not just 10 representative, but randomly selected, high power fields. This difference in approach might explain the underestimation of $\mathrm{H}$-score.

The positive cut off for the EIA is usually $10 \mathrm{fmoles} / \mathrm{mg}$ protein in breast carcinomas. ${ }^{267}$ The positive cut off using the IHA is more contentious and varies from a $\mathrm{H}$-score of 20 ,

Table 2 Frequency of multiplicative quickscores in IHA and ELA result subsets

\begin{tabular}{|c|c|c|c|c|c|c|c|c|c|c|c|}
\hline & \multicolumn{11}{|c|}{ Quickscore $=A \times B$} \\
\hline & 0 & 1 & 2 & 3 & $4-6$ & 8 & 10 & 12 & 15 & 18 & Total \\
\hline \multicolumn{12}{|l|}{ H-score } \\
\hline 0 & 29 & 0 & 0 & 0 & 0 & 0 & 0 & 0 & 0 & 0 & 29 \\
\hline $1-19$ & 2 & 3 & 2 & 0 & 0 & 0 & 0 & 0 & 0 & 0 & 7 \\
\hline $20-49$ & 0 & $1^{*}$ & 0 & 2 & 1 & 3 & 0 & 0 & 0 & 0 & 7 \\
\hline $50-99$ & 0 & 0 & 0 & 0 & 3 & 5 & 5 & 4 & 1 & 2 & 20 \\
\hline $100-199$ & 0 & 0 & 0 & 0 & 0 & 0 & 8 & 10 & 3 & 12 & 33 \\
\hline $200-300$ & 0 & 0 & 0 & 0 & 0 & 0 & 0 & 0 & 0 & 0 & 0 \\
\hline \multicolumn{12}{|l|}{ EIA } \\
\hline$\leq 9$ & 31 & 3 & 2 & 0 & 0 & 0 & 0 & 0 & 0 & 0 & 36 \\
\hline $10-49$ & 0 & $1^{* *}$ & 0 & 1 & 2 & 7 & 5 & 4 & 2 & 2 & 24 \\
\hline $50-99$ & 0 & 0 & 0 & 1 & 2 & i & 5 & 4 & 1 & 2 & 16 \\
\hline $100-199$ & 0 & 0 & 0 & 0 & 0 & 0 & 1 & 5 & 1 & 5 & 12 \\
\hline 200-299 & 0 & 0 & 0 & 0 & 0 & 0 & 1 & 1 & 0 & 3 & 5 \\
\hline $300-399$ & 0 & 0 & 0 & 0 & 0 & 0 & 0 & 0 & 0 & 1 & 1 \\
\hline $400-499$ & 0 & 0 & 0 & 0 & 0 & 0 & 0 & 0 & 0 & 0 & 0 \\
\hline $500-600$ & 0 & 0 & 0 & 0 & 0 & 0 & 1 & 0 & 0 & 1 & 2 \\
\hline Total & 31 & 4 & 2 & 2 & 4 & 8 & 13 & 14 & 4 & 14 & 96 \\
\hline
\end{tabular}

* $=32 ;{ }^{* *}=29$ fmoles $/ \mathrm{mg}$ protein

Negative H-score $\leq 19$.

Negative EIA $\leq 9$ fmoles/mg protein. 
in our laboratory, ${ }^{5}$ to $50-100$ in others. ${ }^{27}$ Bearing in mind our laboratory cut off values, table 2 shows a logical demarcation between the positive and negative EIA and IHA subsets at a multiplicative quickscore $\geqslant 3$. Thirty six breast tumours with negative EIA results ( $\leqslant 9 \mathrm{fmoles} / \mathrm{mg}$ protein) occurred in the 0-2 quickscore subset; no oestrogen receptor negative breast tumours on EIA were reported in the 3-18 quickscore subset whereas 59 of 60 oestrogen receptor positive breast cancers were observed in this classification. Only one of 60 oestrogen receptor EIA positive tumours was present in the $0-2$ quickscore subset. The same results were obtained using a $\mathrm{H}$-score cut off $<20$. The one tumour negative on assessment using the quickscore but positive by IHA (Hscore $=32$ ) and oestrogen receptor-EIA (29 fmoles/mg protein) came from the same patient.

The results also show that there is no difference between adding or multiplying categories $\mathrm{A}$ and $\mathrm{B}$. The additive approach, with a range from 1 to 9, has two disadvantages. Firstly, the additive $(\mathrm{A}+\mathrm{B})$ quickscore does not permit a completely negative result, which occurred in many of our oestrogen receptor negative cases, whereas the multiplicative $(\mathrm{A} \times \mathrm{B})$ quickscore does. Secondly, the range is small for the additive quickscore. Considering the wide range of values within the quantitative methods, the multiplicative quickscore, ranging from 0 to 18 , is preferable. Without prejudicing accuracy, the quickscore approach takes about a quarter of the time taken when calculating the $\mathrm{H}$-score because it dispenses with the need to count individual cells.

G Saccani Jotti was supported in part by the following grants: CNR ACRO Project, CNR Bilateral 94.02482.CT04 and MURST $40 \%$ (Italy). SD and MD were supported by the Cancer Research Campaign.

1 Osborne CK. Receptors. In: Harris JR, Helman S, Henderson IC, Kinne DW, eds. Breast diseases. Philadelphia: JB Lippincott, 1991:301-25.

2 Andersen J. Determination of oestrogen receptors in paraffinembedded tissue. Acta Oncol 1992;31:611-27.

3 McClelland RA, Finlay P, Walker KJ, Nicholson D, Robertson JFR, Blamey RW, et al. Automated quantitation of immunocytochemically localised oestrogen receptors in human breast cancer. Cancer Res 1990;50:3545-50.

4 Barnes DM, Dublin EA, Fisher CI, Levison DA, Millis RR. Immunohistochemical detection of p53 protein in mammary carcinoma. Hum Pathol 1993;24:469-76.

5 Saccani Jotti G, Johnston SRD, Salter J, Detre S, Dowsett $M$. Comparison of a new immunohistochemical assay for oestrogen receptor in paraffin wax embedded breast caroestrogen receptor in parafin wax embedded breast car-
cinoma tissue with quantitative enzyme immunoassay. $\mathcal{I}$ Clin Pathol 1994;47:900-5.

6 Andersen J, Bentzen SM, Poulsen HS. Relationship between radioligand binding assay, immunoenzyme assay and immunohistochemical assay for oestrogen receptors in human breast cancer and association with tumour differentiation. Eur $\mathcal{F}$ Cancer 1988;24:377-84.

7 Kinsel B, Szabo E, Greene GL, Konrath J, Leight GS, McCarty KS. Immunocytochemical analysis of oestrogen receptors as a predictor of prognosis in breast cance patients: comparison with quantitative biochemical methods. Cancer Res 1989;49:1052-6.

\author{
Esbjerg County
Hospital, \\ DK-6700 Esbjerg, \\ Denmark Department \\ of Internal Medicine \\ and Haematology \\ A Siboni \\ T Mourits-Anderson \\ Department of \\ Pathology \\ J Moesner \\ Correspondence to: \\ Dr Anders Siboni, \\ Skolebakken 66,4 ,th. \\ DK-6700 Esbjerg. \\ Denmark. \\ Accepted for publication \\ 1 December 1994
}

\title{
Granulomatous bone marrow inflammation during treatment of chronic myeloid leukaemia with interferon alpha-2b
}

\author{
A Siboni, T Mourits-Andersen, J Moesner
}

\begin{abstract}
A patient with chronic myeloid leukaemia developed bone marrow granulomas during treatment with interferon alpha-2b. Some granulomas had necrotic centres and giant cells and there was marked eosinophilia surrounding them. The granulomas disappeared when the interferon treatment was discontinued. Mycobacteriosis was ruled out. The most likely explanation for the granuloma formation was drug hypersensitivity.

(f Clin Pathol 1995;48:870-880)
\end{abstract}

Keywords: interferon, bone marrow granulomas.

The treatment of chronic myeloid leukaemia with interferon alpha-2b (IFN- $\alpha-2 b$ ) prolongs survival if cytogenic response occurs: in one study, partial cytogenetic response and temporary morphological remission was seen in $46 / 71$ patients. ${ }^{1}$ Interferon alpha is used in the treatment of both infectious and malignant diseases. ${ }^{2}$ One of its mechanisms of action may be inhibition of formation of new blood vessels (antiangiogenesis) in tumour areas. ${ }^{2}$

Bone marrow necrosis at blast transformation of chronic granulocytic leukaemia treated with interferon has been described by Kendra $e t ~ a l,{ }^{3}$ but the formation of bone marrow granulomas during the treatment of chronic myeloid leukaemia with IFN- $\alpha-2 b$ has not been described before.

\section{Case report}

A 43 year old man was referred in August 1992 because of a leucocyte count of $89 \times 10^{9} / 1$. Bone 